Article

\title{
A Data Driven Approach to SAR Data Focusing
}

\author{
Cataldo Guaragnella ${ }^{(1)}$ and Tiziana $\mathrm{D}^{\prime}$ Orazio $^{(2)}$ \\ (1) DEI - Department of Electrical and Information Engineering, Politecnico di Bari, Bari, Italy, \\ cataldo.guaragnella@poliba.it \\ (2) STIIMA - Institute of Intelligent Industrial Technologies and Systems for Advanced Manufacturing, \\ CNR - Italian National Research Council, Bari, Italy, tizianarita.dorazio@cnr.it \\ * Correspondence: cataldo.guaragnella@poliba.it; Tel.: +39-080-596-3655
}

\begin{abstract}
Synthetic Aperture RADAR (SAR) is a radar imaging technique in which the relative motion of the sensor is used to synthesize a very long antenna and obtain high spatial resolution. Standard SAR raw data processing techniques assume uniform motion of the satellite (or aerial vehicle) and a fixed antenna beam pointing sideway orthogonally to the motion path, assumed rectilinear. Despite SAR data processing is a well established imaging technology that has become fundamental in several fields and applications, in this paper a novel approach has been used to exploit coherent illumination, demonstrating the possibility of extracting a large part of the ancillary data information from the raw data itself, to be used in the focusing procedure. In this work an effort has been carried out to try to focus the raw SAR complex data matrix without the knowledge of any of the parameters needed in standard focusing procedures as Range Doppler (RD) algorithm, $\Omega-K$ algorithm and Chirp Scaling (CS) algorithm. All the literature references regarding the algorithms needed to obtain a precise image from raw data use such parameters that refer both to the SAR system acquisition geometry and its radiometric specific parameters. In [22], authors introduced a preliminary work dealing with this problem and able to obtain, in the presence of a strong point scatterer in the observed scene, good quality images, if compared to the standard processing techniques. In this work the proposed technique is described and performances parameters are extracted to compare the proposed approach to RD.
\end{abstract}

Keywords: SAR system; efficient focusing of SAR data; Inverse problem; radar theory; remote sensing; SAR data focusing; phase shifts; satellite trajectory; spatial resolution; synthetic aperture radar; Geometry; Satellites; Ancillary Data; Singular Value Decomposition; Blind deconvolution; Signal Processing; Parameter estimation; Algorithm; Imaging; Phase estimation; Phase compensation; Computational modeling; Image resolution; Synthetic Aperture

\section{Introduction}

The Synthetic Aperture Radar ([1][2][3][4])can acquire very high resolution images of the inspected area using high bandwidth of the transmitted coherent illumination signal by means of an accurate processing of the ground received returns. In a standard structure, the system is composed of a platform (i.e. airborne or satellite) using the same antenna both for the transmitting and receiving phases; the target scene is repeatedly illuminated with pulses of radio waves. The signals echoes are received in the band of the transmitted pulse along the satellite track at different positions, converted to an intermediate frequency channel and IQ sampled so that a baseband processing procedure can allow the synthetic aperture processing algorithm, obtaining the equivalent return of a very narrow antenna beam. Due to physical limitations, it is not possible to manufacture an antenna of long length and mount it on an airborne platform, and this is particularly true for small and cheap unmanned Aerial Vehicles (UAV) so that the narrow beam in the along track direction (Azimuth) is synthesized from data. Three main algorithms are available to obtain such high quality images, namely Range Doppler ([6]) algorithm, $\Omega-K$ ([5]) algorithm and Chirp Scaling algorithm. All such algorithms 
require precise geometric acquisition parameters and radiometric parameters. Such parameters are always available as a side documentation of each acquired image.

Fig.1 reports the acquisition geometry of a SAR system. The synthesis procedure in focusing the acquired data is carried out by coherent integration. Each target on the ground contributes to the radar return on several subsequent transmitted pulses. In SAR two main directions are important to focus the data: slant range direction, in which transmitted pulses travel, and Azimuth direction, i.e. the direction of sensor movement. The precise knowledge of the geometry of the acquisition allows to add in phase each contribute of the single point scatterer on the ground to obtain the focused image. The wider the beam, the less the detail acquired by any return, but the larger the integration size of the track contribution to synthesize the image, so that the azimuth radar resolution is not theoretically bounded. The practical azimuth resolution is limited by the PRF choice (the Pulse Repetition Frequency of transmitted pulses used for coherent illumination of the target area), i.e. the azimuth sampling frequency.

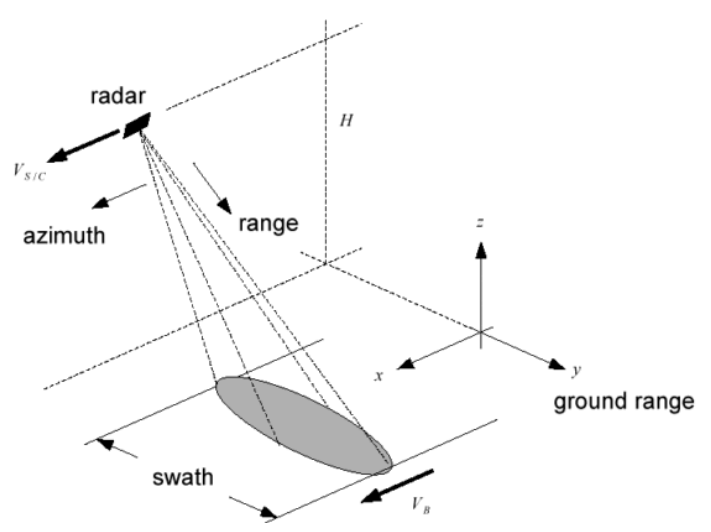

Figure 1. Sar Data acquisition geometry

In several cases, due to imprecise knowledge of the satellite or aerial vehicle acquisition geometry or due to the presence of motion in the scene (ships, cars, etc.) defocusing of the obtained image appears so many authors addressed the problem of a post processing procedure able to exploit the residual correlation present in data to perform accurate focusing of the image. Motion compensation is very important to help achieving high resolution in SAR image. The high frequency phase error that may be present on the focused image can be compensated through Inertial Measurement Unit (IMU) and Global Positioning System (GPS) side information. However, the need to measure and add such information to the ancillary data complicates the burden on any motion compensation system. In this cases SAR autofocus algorithms ([11][12][13][14][15]) are used to solve the problem in a blind mode. SAR autofocusing algorithms are categorized into three types: sub-aperture-based algorithms, prominent point-based algorithm and metric-based autofocus. Most of the traditional autofocus algorithms assume there are strong scatters in the scene. Compared to the other conventional autofocus methods, the metric-based methods can work well without prominent points but deal with an already focused image.

The defocused image can be considered as the perfect focused image convoluted with the pointed spread function (PSF) caused by the phase error. Only recently and with the advent of lightweight and cheap SAR systems and UAV and drones, the problem of high cost of the system has pushed research to find new solutions in the development of such systems that imply in some cases the development of new techniques trying to focus the acquired raw data matrix using a subset of the ancillary data parameters and in presence of strong geometry anomalies that occur in such cases ([16][17][18][19][20][21]). As the availability of parameters needed to extract has always been guaranteed no author, at our knowledge, tried to estimate the reference function to be used as the focusing operator from the data itself and develop a completely blind focusing procedure. 
While all the available algorithms to solve the SAR data focusing problem are model driven, as they use ancillary parameters information to model the inverse problem in radar soil backscatter, in this paper a data driven approach is proposed to develop a totally blind SAR data focusing is presented, able to obtain good image quality working on the complex SAR raw data matrix in absence of any information about the sensor. The proposed approach at the state of the art works well in the presence of a strong point scatterer in the scene.

The main idea of the proposed approach is to exploit all the inherent information intrinsically stored in the data itself to extract the focusing reference functions to be used in a $\Omega-K$ or RD algorithm to obtain the Single Look Complex of any SAR sensor without even knowing important ancillary data parameters, needed by all the SAR focusing processors, such as the distance at the center of the beam, the radar sampling frequency, the transmitted chirp bandwidth, the chirp rate and the chirp duration, the radar wavelength, the PRF, the sensor speed and the off nadir angle, used in data acquisition.

The proposed approach has been tested on several images of ERS raw data, made accessible for the scientific purpose from the Italian Space Agency (ASI) and the cross comparison with the state of the art focusing algorithm is carried out. Preliminary results seem to indicate a good accordance to the standard focusing of obtained images with respect to the officially distributed ones. The proposed approach can reveal interesting and convenient in several application fields such as local monitoring zones made by SAR systems carried by small lightweight and low cost aerial unmanned vehicles. Modern hardware technology permits to reduce the dimensions and weights of SAR systems into small and cheap flying platforms that can be conveniently used with low cost unmanned aerial vehicle (UAV) platforms and flying drones. High-resolution microwave images of the observed scene can be obtained under various environmental conditions. Thus, UAV-SAR attracts growing interest in recent years ([17]). The possibility of developing commercial low cost systems is anyway still limited by the complication of the development of SAR due to the precise need of mission parameters to obtain good quality images. Such parameters are very unstable for this kind of applications; furthermore the knowledge of all the mission parameters introduces a complication in the system in storage of ancillary parameters files, increasing their cost.

With respect to the reference [22] in which authors have preliminary proposed the blind technique to focus SAR data in the presence of a point scatterers in the scene, in this paper a more complete discussion about the quality of the focused image is carried out. To define the resolution in the range and azimuth directions, point scatterers responses are extracted from the image and azimuth and range cuts are compared with range doppler focusing of the same image. Also, an interferometric pair has been processed and the interference fringes have been extracted to show the good performance and phase stability of the proposed technique.

\section{SAR data acquisition}

SAR normally use chirp transmitted pulse. The radar moves along the track in the azimuth direction while transmitting repeated chirp signals with a given PRF in the direction orthogonal to the satellite trajectory (slant range). The received samples of the radar return are acquired along the slant range direction and each recorded data vector represents a row of a sequentially stored matrix of complex data (raw data matrix). The chirp signal is a linearly frequency modulated (FM) signal spanning the bandwidth of the radar; its peculiarity is the ability of conveying high energy of the transmitted long pulse duration without the limitation inherent in geometric resolution because the use of a matched filter at the receiver end allows to recover a high peak signal to noise ratios and a resolution in distance directly related to the chirp signal bandwidth ([1], [2], [3]). Due to the many return contributions at each given instant of the recorded file (the superposition of returns of all the points on the ground within the physical antenna footprint located on the Earth at the same distance from the sensor), the acquired raw data matrix assumes the aspect of a complex random noise with a gaussian distribution. The problem of SAR data focusing is commonly known as an inverse problem, 
where the deconvolution algorithm that permits to obtain the focused image makes recourse to the definition of the correlation operator to be used on raw data, precisely known if several mission parameters are known; such parameters are made available, for each RAW data product, in the form of an ancillary information file. As the SAR algorithm is linear, the deconvolution implementation can be described observing a single point target at the center of the scene (or within) and extract all the useful information from geometrical considerations.

\section{Transmitted Chirp}

At every position along the sensor track of a perfectly absorbing surface where a single strong point scatterer is placed, a return of the scatterer to the chirp is recorded. This signal is acquired along the track in all the point until it remains within the azimuth antenna beam pattern. The antenna gain changes and assumes its largest value when the scatterer is exactly at the center of the antenna pattern while the intensity of the received return is tapered for different position of the sensor along the track.
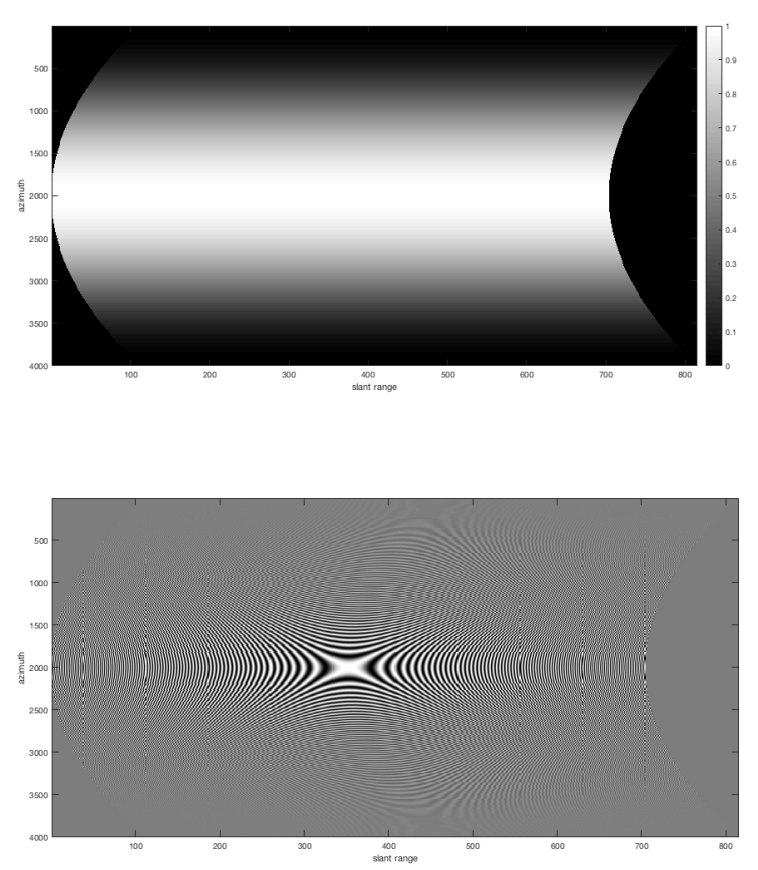

Figure 2. Simulated response of a point scatterer: Magnitude and real part

Fig. 2 reports the magnitude of the response of a simulated single point scatterer in an ideal acquisition SAR system. What is clearly visible in the figure is both the degradation of the response for azimuthal position displaced from the center beam and the phenomenon known as range migration or range walk, that is the displacement, in the slant range direction, of the first sample of the radar return as the sensor moves along the track. In the figure is also represented the real part of the simulated raw data.

\subsection{Range Migration and Azimuthal chirp}

If the raw data matrix in Fig. 3 is read in the vertical (azimuth) direction, due to the range migration and delay in received chirp response of the single point scatterer on the ground, the azimuth response reveals to be a chirp signal too, amplitude modulated by the antenna beam pattern in azimuth (see Fig.3). The state of the art focusing algorithms obtain high resolution images from 
SAR raw data matrices basing on precise knowledge of the data acquisition geometry and SAR system parameters. The mostly used SAR focusing algorithms are RD and $\Omega-K$. Also a modified version of the focusing algorithm is the Chirp Scaling (CS). In the Range-Doppler approach, the focusing algorithm can be decomposed easily in two different parts: first focusing in the range direction is carried out by correlating the received raw data rows with the range reference function (i.e. the transmitted chirp signal); once obtained the range focused image, focusing in the azimuth direction takes place normally in two steps: first, the range migration compensation is applied (Range Cell Migration Compensation), then the azimuth compression takes place using a geometrically constructed phase history of the received signal.

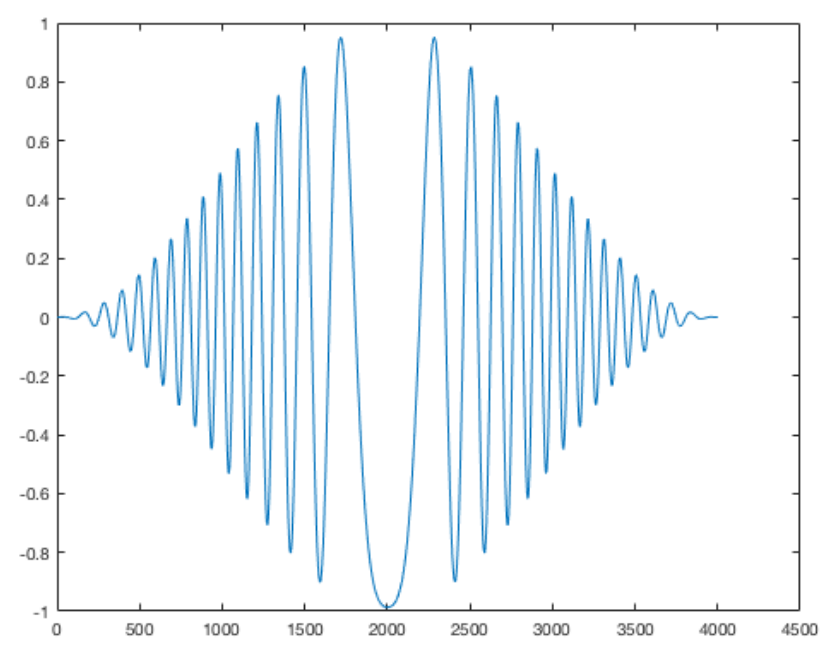

Figure 3. Azimuth chirp, real part

In a real scenario, several other distortions can take place, due to non ideal acquisition geometry. The presence of a shift in the doppler centroid requires its estimation and compensation; such phenomenon is generally due to relative motion of Earth ground point with respect to the sensor trajectory or to the satellite attitude deviations with respect to theoretical assumed one. Several refinement algorithms have been presented in the scientific literature and are part of the focusing algorithm actually available and used to produce official data ([9], [10], [11]). Also efficiency has always been addressed in the performances evaluation of a focusing algorithm, nowadays overcome by the high computational capacity of existing hardware that makes this problem less severe than once.

\section{Extracting information from SAR data}

Several information about the SAR data format can be extracted from the acquired data. In this section all the useful information that can be extracted from the data itself will be addressed and the required procedures to extract the information will also be presented.

\subsection{Blind SAR data Focusing Algorithm}

The acquired data, as discussed, is the result of backscattering contribution of the ground at the SAR frequency. As a coherent illuminating source is used, the received data refer to several observations of the same scene taken in different points along the satellite trajectory; each return should contain, for several pulses in the sequence, roughly the same information so that the exploitation of coherence of the received signal can be attempted. The radar transmitted pulses are stable in time: all the received returns show a strong azimuth correlation. This hypothesis allows to use some correlation-based algorithm to extract useful information from data. The use of Singular 
Value Decomposition (SVD) ([6]) technique can give us information about the reference functions to be used to focus the image.

\subsection{SVD - Signal Processing}

Singular Value Decomposition ([7]), in its economy formulation, is a standard algorithm able to decompose a given rectangular matrix into the product of three matrices, $U, S$ and $V$ as schematically depicted in Fig. 4.

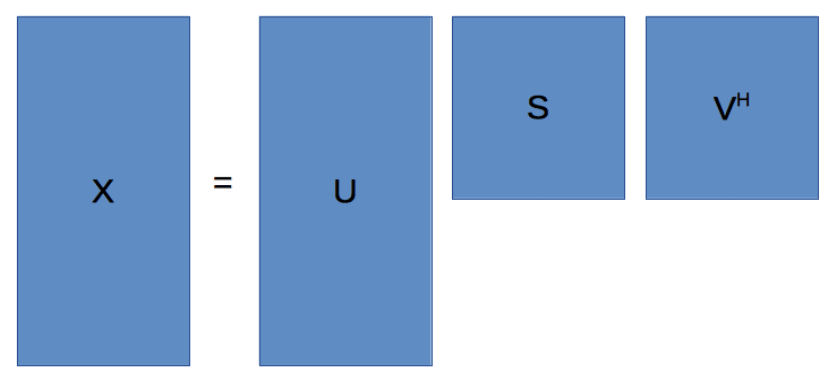

Figure 4. Schematic description of the SVD decomposition

$X$ is the data matrix of size $M \cdot N, U$ and $V$ are orthonormal matrices; $U$ has the same size of the matrix $X$ while $S$ is a real valued diagonal matrix of size $N$ and $V$ is a complex orthonormal square matrix of size $N$, where:

$$
\mathbb{U}^{\mathbb{H}} \cdot \mathbb{U}=\mathbb{I}_{\mathbb{N}}
$$

and

$$
\mathbb{V}^{\mathbb{H}} \cdot \mathbb{V}=\mathbb{I}_{\mathbb{N}}
$$

$S$ is the matrix containing the Singular Values of the matrix decomposition, sorted along the diagonal from the highest value to the lowest. In Analytical form, The SVD decomposition can be written simply as:

$$
\mathbb{X}=\mathbb{U} \cdot \mathbb{S} \cdot \mathbb{V}^{\mathbb{H}}
$$

where the superscript $(\cdot)^{H}$ represents the transpose and conjugate operator (Hilbert operator). If a right multiplication for matrix $\mathrm{V}$ of both terms in (3) is applied, it can be usefully restated in another form:

$$
\mathbb{X} \cdot \mathbb{V}=\mathbb{U} \cdot \mathbb{S}=\mathbb{E}
$$

The $V$ matrix is the matrix mixing columns of the data matrix $\mathrm{X}$ to obtain $\mathrm{E}$, an orthogonal matrix. The right multiplication of the matrix $U$ with $S$ only scales the vector columns in $U$ non affecting the orthogonality property. E is a matrix containing columns obtained from the linear mixture of the columns of the data matrix. The columns of the original data matrix can be considered as different realizations of a given process, so that this orthogonality and the hypothesis of zero mean for the signal time series permits the separation of the data matrix in uncorrelated signals. As a simple example if the SVD decomposition $X$ of two columns requires the right singular vector matrix $V$ an orthonormal matrix of size 2. The $V$ matrix in this simple case represents a complex Gibbs rotation matrix. In this very simple case, the operation carried out by the decomposition becomes clear:

$$
\left[\begin{array}{ll}
\overrightarrow{x_{1}} & \overrightarrow{x_{2}}
\end{array}\right] \cdot\left[\begin{array}{cc}
c & s \\
-s & c
\end{array}\right]=\left[\begin{array}{ll}
\overrightarrow{e_{1}} & \overrightarrow{e_{2}}
\end{array}\right]
$$


The E matrix is thus obtained as a simple linear combination of columns of $\mathrm{X} . \mathrm{V}$ is the Gibbs rotation matrix and:

$$
|c|^{2}+|s|^{2}=1
$$

In a geometrical representation we can consider $c$ and $s$ parameters able to scale, rotate and phase shift vectors they multiply so that their sum and difference mixtures gives the two orthogonal vectors in $E$. The modula of such vectors represent the singular values and one can easily demonstrate that such values are proportional to the estimates of the standard deviation of the resulting signals in $E$. As in $E$ the two transformed vectors are independent, the mostly correlated information in the two signals in $X$ is transferred to the first singular vector in $E$, while the remaining part is in the second. When applied to a multicolumn matrix, this procedure tends to accumulate all the strongly correlated information on columns in the first left eigenvector, so that it contains the information in on the image columns in the azimuth direction. The coherent illumination due to the transmission of the chirp produces very correlated information; in particular, in the azimuth direction the correlated information is the doppler phase history due to the scanning process. The expected result is that the first left singular vector should closely be related to the doppler history of the SAR system.

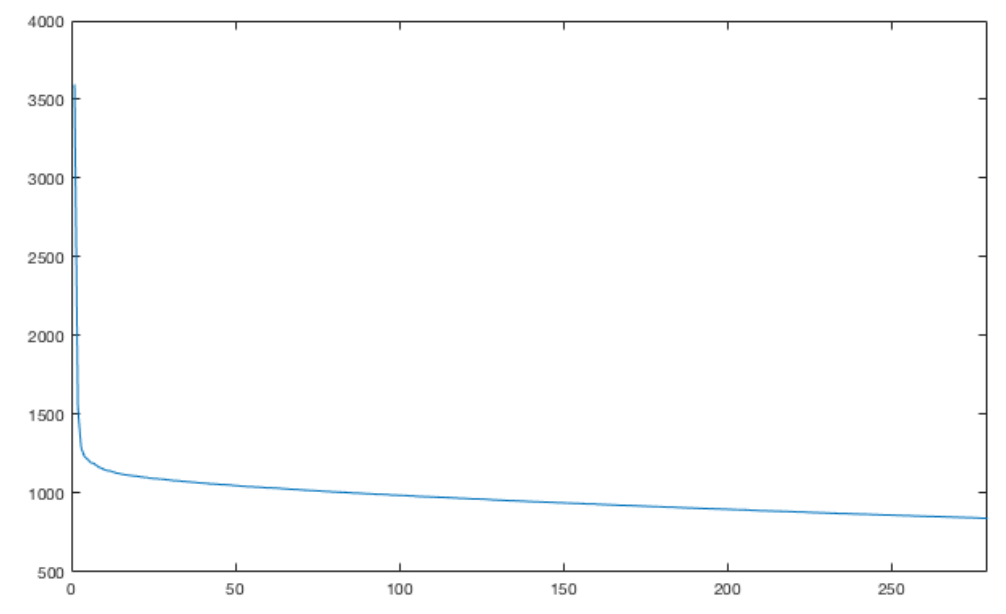

Figure 5. Singular Values for the SAR raw data matrix decomposition

Also, accordingly to the SVD decomposition scheme, the right singular vectors contain the mixing coefficient able to orthogonalize the raw data matrix. This information is closely related to the transmitted information in the slant range direction. All the rows in the data matrix contain the same information, i.e. the transmitted chirp, delayed and phase shifted of an amount depending on the SAR geometry. The data are strongly correlated, so the SVD decomposition will store automatically in the rotation matrix $V$ all the coefficients able to phase shift the subsequent transmitted chirps in a way that their sum convey the most important part of the global transmitted energy of the pulse. The coefficient must then be closely related to both the geometrical properties of the SAR acquisition and the characteristics of the transmitted chirp. This consideration will be illustrated in the next paragraph.

Basing on these observations, a simple and direct scheme to obtain a good focusing of the raw data in a blind mode has been devised.

\section{Experimental results}

The SVD decomposition is then applied as a test of the proposed approach to several ERS raw data matrices without any knowledge of the mission ancillary data. The plot of the diagonal elements 
of matrix $S$ is a simple description of how the energy of the orthogonalized signals is distributed. This gives a quick description of how efficient was the separation of signals into uncorrelated components. Fig.5 shows the plot of singular values for the ERS data matrix.

A large part of the energy in the data matrix is concentrated in the first singular value, clearly stating that the first left singular vector (i.e. the first column of matrix $U$ ) should contain the orthogonal signal with maximum energy in the data. Fig.7 b) represents the real part of this singular vector and, as it can be seen, it is clearly similar to the azimuth chirp, hence showing also the antenna beam pattern intensity modulation. From the simple observation of the first left singular vector of the matrix $U$ two informations can be extracted: the antenna beam pattern in azimuth and the phase history of the azimuth reference to be used in the focusing procedure.

Thresholding can be applied to define the useful phase history and estimate the antenna beam pattern. Also, the estimate of the doppler centroid can be carried out measuring the azimuth position of the lowest instantaneous frequency with respect to the peak value of the azimuth reference function, representing the antenna beam pattern central position. Considering the matrix product of the SVD decomposition, to extract the first left singular vector of matrix $U$, the mixing coefficients to be used are the ones in the right first singular vector of matrix $V$. This signal contains the complex coefficients needed to re-phase and add the received chirp signal in the raw data matrix. It is interesting to verify what such coefficients are. Fig. 6 a) represents the real part of the first column of the matrix $V$. The proposed approach is thus simple and direct and allows to extract useful information to focus the received data.

It should be pointed out that the possibility of obtaining good estimates of the range and azimuth chirp responses is due to the clear presence of a point scatterer with high signal to noise ratio that conveys a large part of the data matrix energy in a restricted part of the data matrix, allowing good estimates. Once known parameters of the azimuth and range histories, focusing can become simple and can be carried out with either RD or $\Omega-K$ algorithms. In this paper the frequency approach has been used to obtain the focused image. In the subsequent subparagraph the details of the proposed algorithm are given.
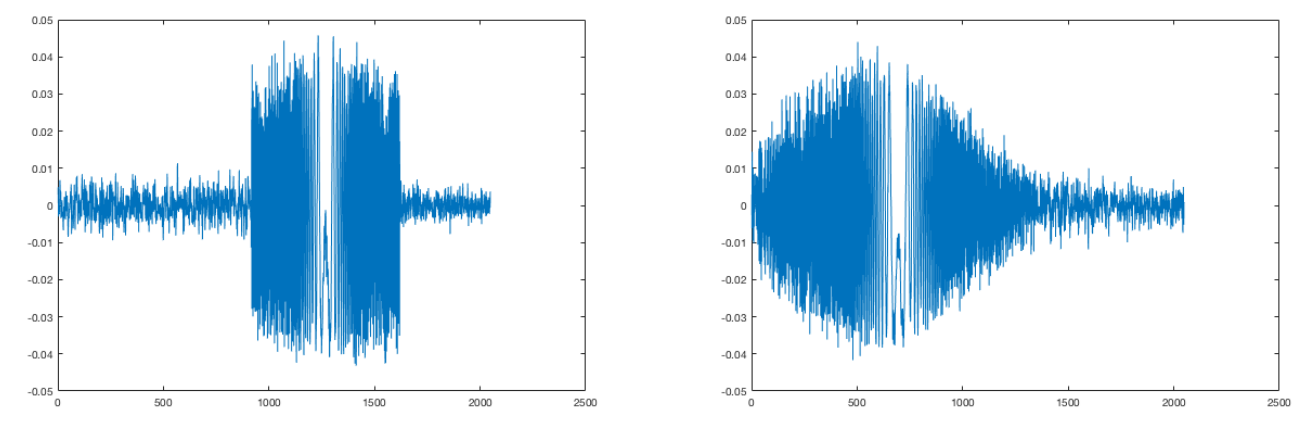

Figure 6. Raw range and azimuth reference functions as extracted from the SAR raw data bi SVD processing. For the azimuth reference is evident the antenna azimuth beam pattern shaping. A moderate attenuation pattern in the range direction is visible also for the range reference function

\section{B-SAR - Blind SAR Data focusing Algorithm}

From the matrix $U$ two informations can be extracted: the antenna beam pattern in azimuth and the phase history of the azimuth reference to be used in the focusing procedure. Thresholding can also be applied to define the useful phase history and estimate the antenna beam pattern. Also, observing the azimuth position of the lowest instantaneous frequency with respect to the azimuth antenna beam center the estimate of the doppler centroid can be obtained. Fig. 6 a) and b) show that the obtained information is noisy so that a more accurate derivation of the azimuth and range histories can be carried out to obtain a clean focusing reference function. 
To select the proper length of the range chirp history and reconstruct a clean reference in range, simple thresholding is used as the reference signal is much higher than the background noise. In this case the selected range time duration by the choice of a threshold at $10 \%$ of the signal peak value has revealed efficient. The estimated length of the range chirp was 703 samples. To reduce the influence of noise, the unwrapped phase of the range chirp has been used in the LMS estimation of the parabolic phase in the construction of the range chirp to be used in the focusing algorithm.
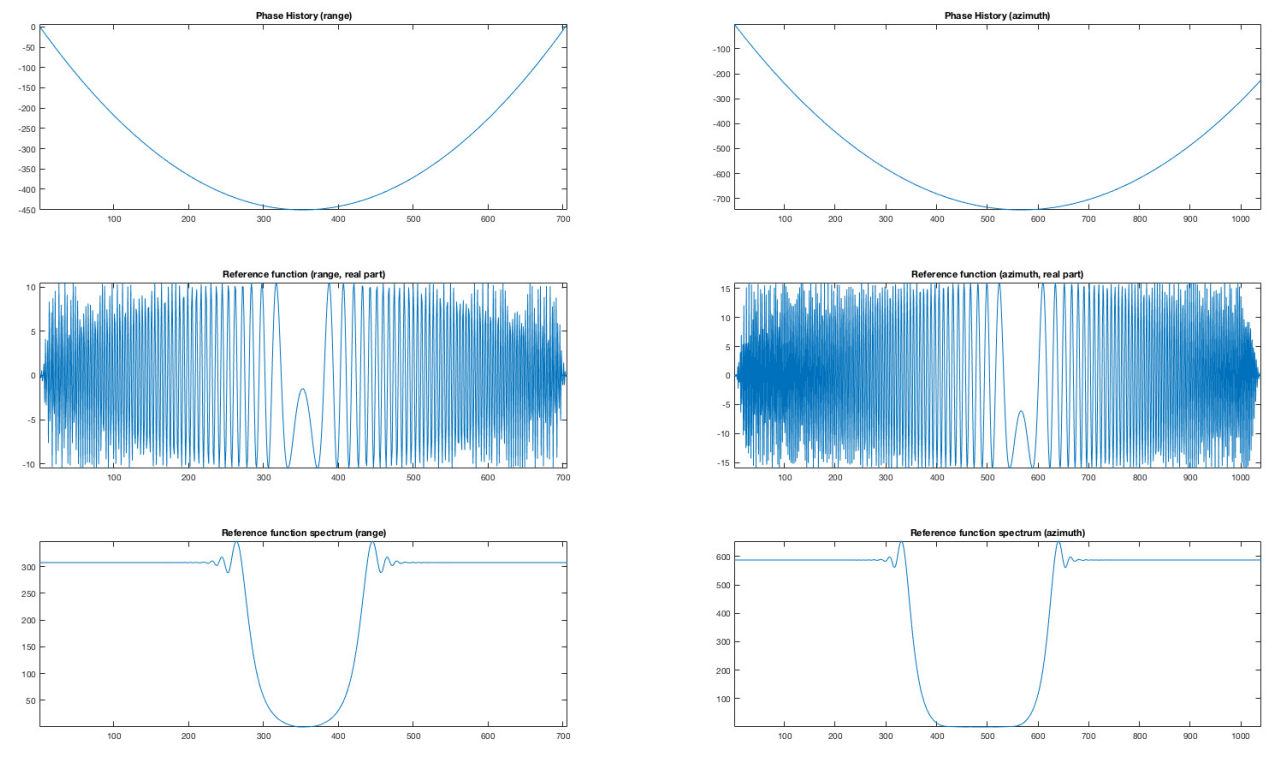

Figure 7. Range and Azimuth references to be used in the focusing procedure. Left range, right azimuth. The three rows present extracted phase histories, the real parts of the reference functions and their spectra

The same procedure was carried out for the azimuth chirp, with a slight more care: the antenna beam pattern estimated in this way is not always effective due to the growing attenuation and the joint influence of azimuth and range beam patterns with the slant range and the possible presence of extended strong scatterers that can reduce the quality of the estimated pattern. Also, it is not clear where the azimuth phase history should be stopped. The main objective is to limit the phase history in a proper way to avoid azimuth aliasing.

The proposed approach uses the phase unwrapping of the azimuth history. The cut of the important information was obtained also by thresholding with respect to the antenna beam pattern maximum, occurring at the signal peak position and selecting the phase history in a generally asymmetric interval around the peak. The limitation of this interval was chosen as the $10 \%$ of the estimated peak of the beam pattern. Also, tapered tails are used to avoid aliasing effects on the focused image, both in the range and azimuth reference functions.

Once obtained via LMS the references of azimuth and range histories (see Fig.8), we can consider ready to focus the image. A block diagram of the complete algorithm is reported in Fig. 8. In ??, the focused ERS image obtained with the proposed algorithm (B-SAR) and the focusing obtained by a standard Range-Doppler SAR processor are compared.

\section{Semi Quantitative Evaluation of focusing performances}

In this paragraph the comparison of the range and azimuth cuts of the proposed B-SAR algorithm in comparison with RD is carried out. In particular, in figure 10 and 11 the range and 
azimuth cuts and the contour shaping of the same point scatterer on respective images obtained focusing the raw SAR data matrix are presented, showing how the range focusing obtained by the proposed procedure seems to adhere more precisely to the theoretical one than the RD focusing.

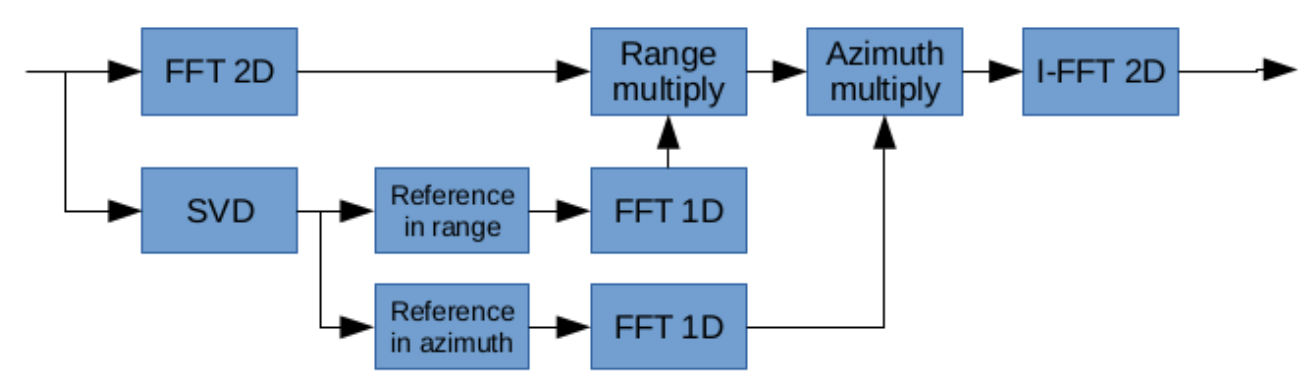

Figure 8. B.SAR Data Processing block diagram
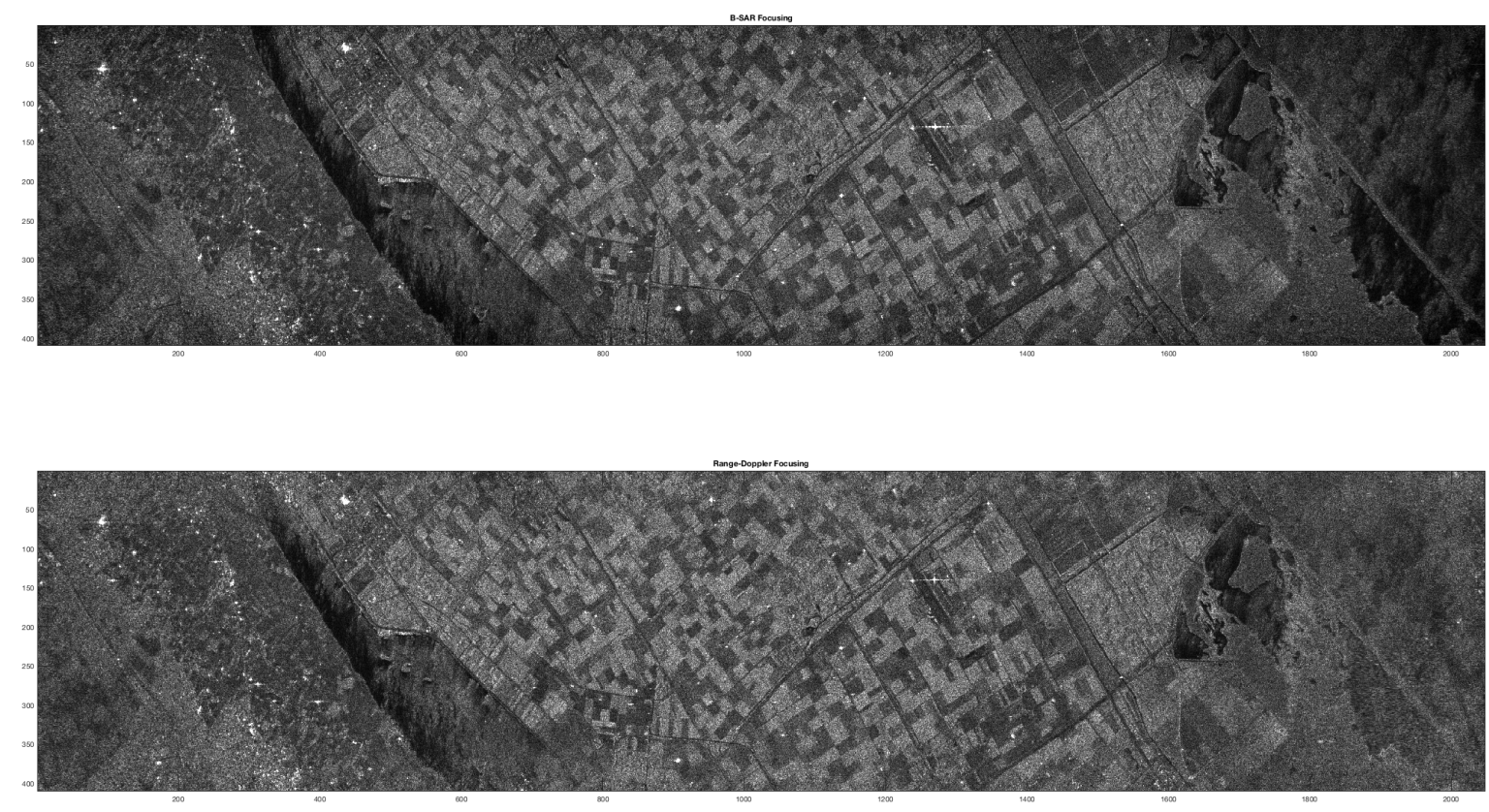

Figure 9. Sample of focused image with the proposed algorithm (upper) compared with the standard focusing obtained via Range Doppler algorithm (lower). ERS 1 - Matera

On the other side, at a lower resolution achieved by RD in the range direction, a higher rejection of side lobes is obtained, showing that the clean aspect of the focused image is due to a higher smoothing of range lines. Figure 12 shows the reference functions (first left and right singular vectors) of the SVD procedure carried out on the complex raw data matrix. 

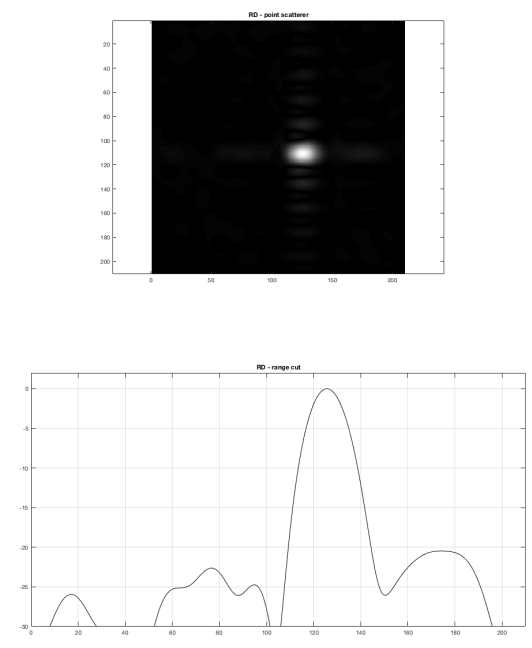
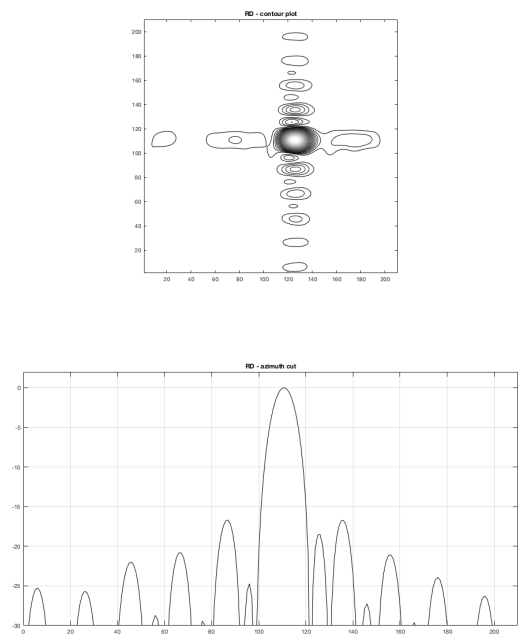

Figure 10. Range Doppler algorithm. Point scatterer image, contour plot and range and azimuth cuts
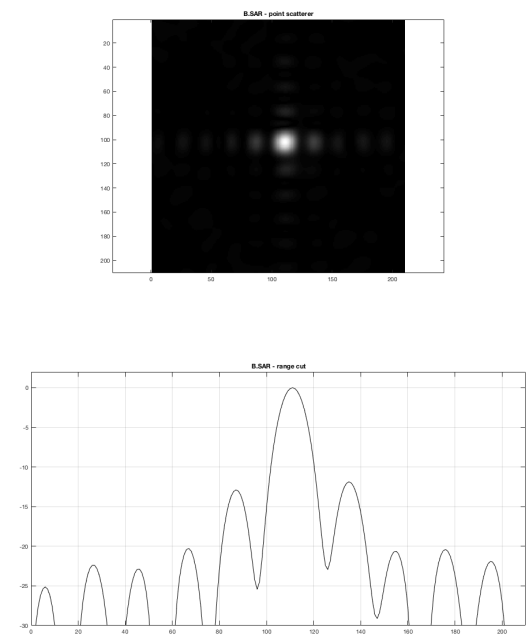
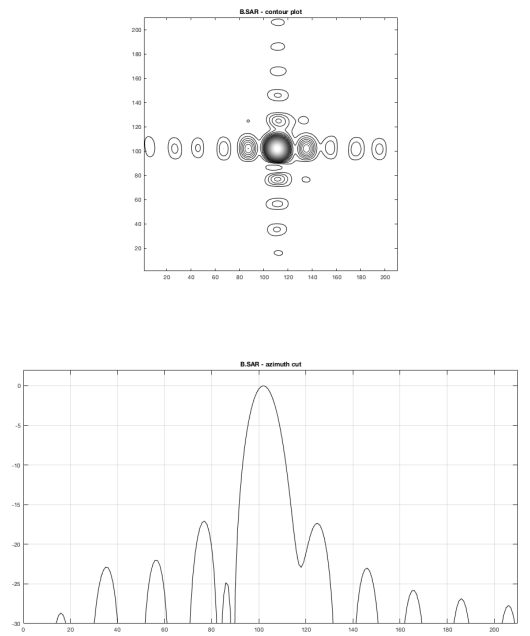

Figure 11. B-SAR algorithm. Point scatterer image, contour plot and range and azimuth cuts

\subsection{B-SAR Phase stability}

The possibility of using the proposed algorithm in several applications in the field of Earth observation, interferometry and multi-temporal interferometry should be a goal to pursue even when non mission parameters are known. To this goal, phase stability of the proposed approach for blind SAR data focusing should reveal stable in phase. To address the phase stability the interferometric image has been computed in a tandem pair image of ERS1-2 pass over the Fucino region in Italy. Fig. 13 shows one of the focused images of the tandem pass pair, while figure 14 shows the (5 looks, slope corrected) interferometric image obtained by B-SAR focusing algorithm with superimposed the intensity image. A close correspondence between the flat zones in the valley with the smooth variations of the phase seem to assess the good behavior and the phase stability of the proposed processing technique. 
The superposition of SAR image and interferometry fringes is reported in the last figure showing a good adherence to the zones of the region imaged. In particular, very smooth interference fringes are evident on the flat land region among hills around.
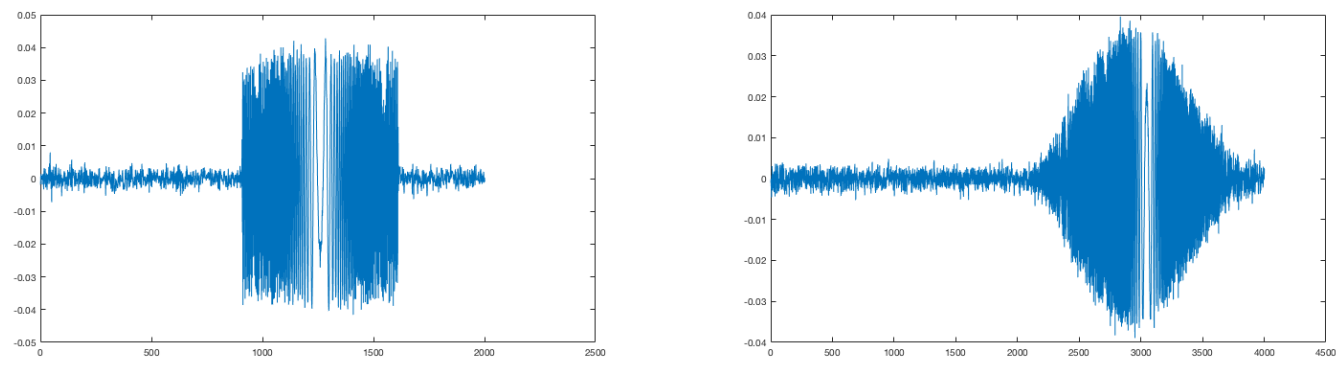

Figure 12. The reference functions (real parts) of the SVD procedure carried out on the complex raw data matrix: the quasi constant amplitude of the range chirp and the beam shaped returns in the azimuth direction are clearly visible

\section{Discussion, limitations and future developments}

In this work the SVD decomposition has been used to extract correlated information from SAR raw data on scenes where a strong point scatterer is present. The use of the SVD is a sufficient information allowing the development of a simple and direct procedure to focus the acquired data without the need of information about the sensor attitudes, path and SAR system parameters. The proposed algorithm, at the state of the art, is sufficient to obtain a fair focused image. The appearance of the focused image obtained with the proposed algorithm is comparable with standard RD focusing, as shown in Fig.9. To assess the performances of the proposed approach, point scatterer responses have been compared between the RD and B-SAR focused images, showing a pretty good correspondence.

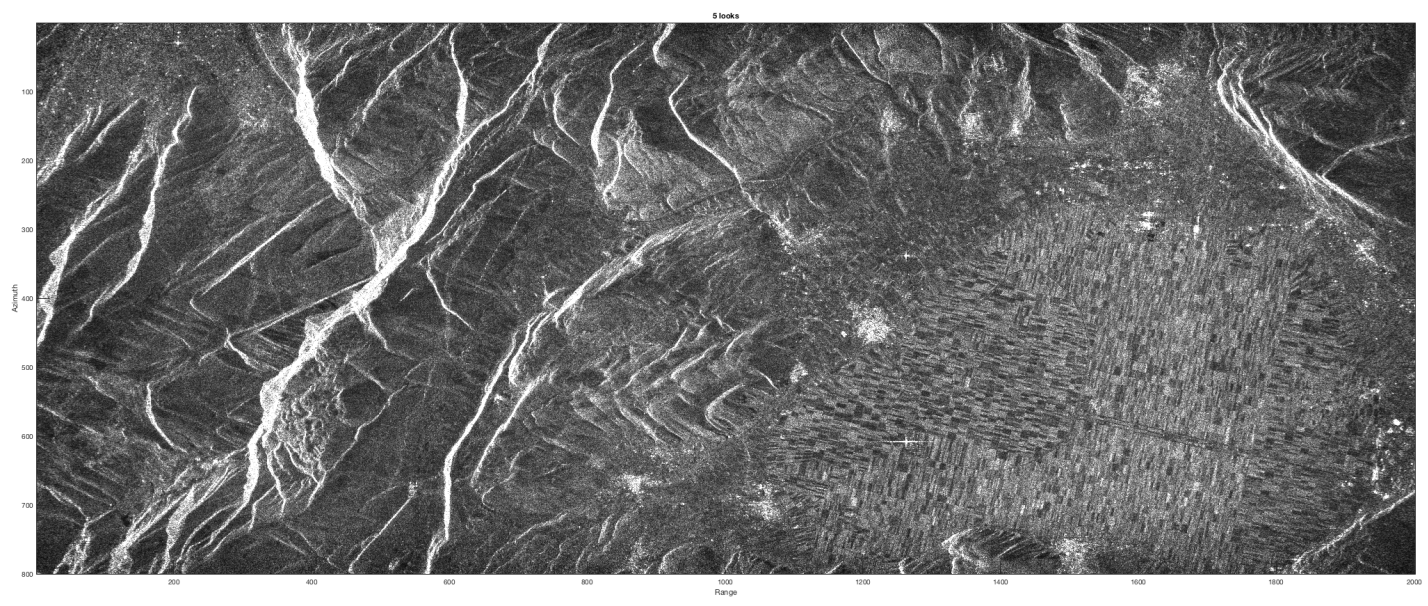

Figure 13. B-SAR focusing. Caramanico site, Fucino Valley, Italy

Also the problem of phase stability of the algorithm has been addressed, computing the interference fringes corresponding to a ERS 1-2 tandem mission, showing also good adherence o the specific local orography. The main limitations of the proposed algorithm lyes in its need of the presence of a strong point scatterer in the imaged zone. This limitation is payed back by its simplicity and the lack of need of the ancillary parameters file in the focusing procedure, aspect that simplifies both the processing and the development of simple and cheap SAR systems to be used in local monitoring also with the recourse to simple aerial unmanned vehicles like drones. 
Also, the possibility of blind focusing SAR raw data, here addressed only in the presence of a point scatterer (e.g. a corner reflector or a transponder), in the general case of SAR stripmap data focusing represents the field of application for future work.

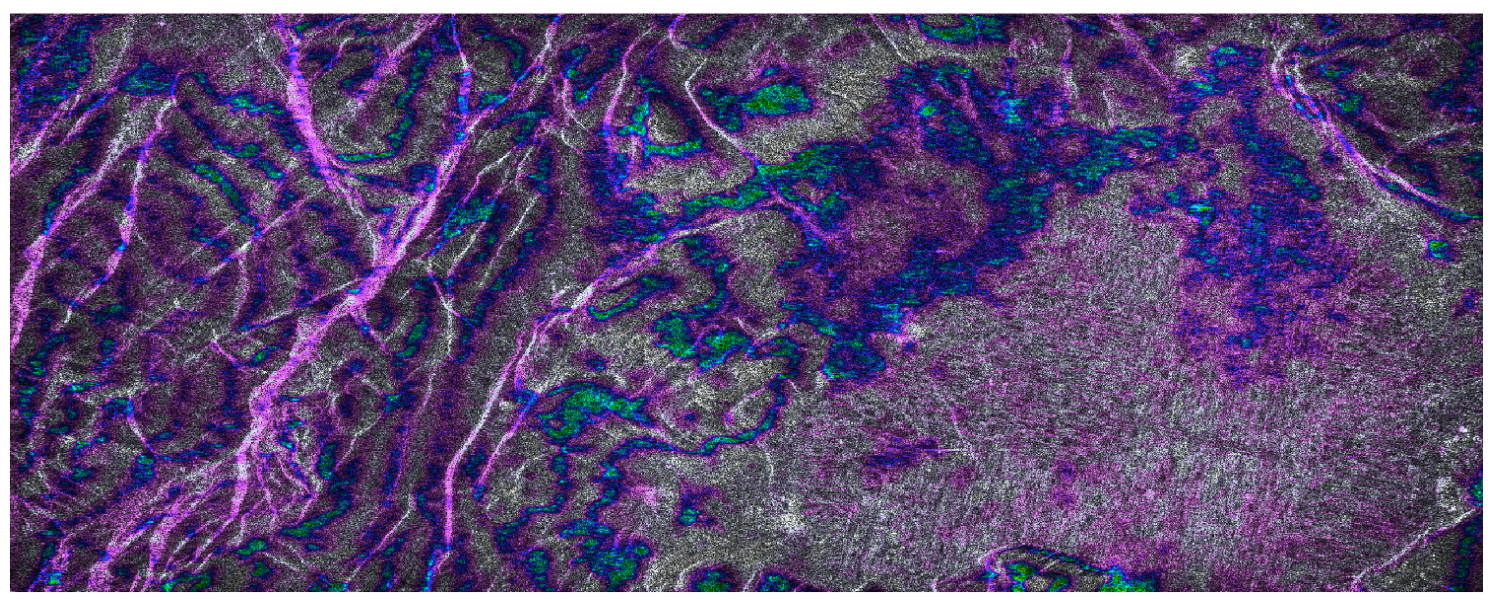

Figure 14. B-SAR, 5 looks, slope corrected interferometric image obtained by B.SAR focusing algorithm with superimposed the intensity image. Caramanico site, Fucino Valley, Italy.

\section{Conclusions}

Fair maps can be obtained by focusing SAR stripmaps raw data with the proposed algorithm. Obtained results are close to the standard images given by the official space agencies. The proposed algorithm, developed in Matlab, is distributed under the Noncommercial - Share Alike 4.0 International Creative Common license by the authors.

\section{References}

1. Ridenour, L. N., Radar System Engineering, McGraw-Hill Book Co.,New York, 1945, or Boston Technical Lithographers, Lexington, Mass., 1963.

2. Skolnik, M. I., Introduction to Radar Systems, McGraw-Hill Book Co., New York,1962.

3. Klauder, J. R., Price, A. C., Darlington, S., Albersheim, W. J., The Theory and Design of Chirp Radars," Vol. 39, No 4, Bell System Technical Journal, July 1960.

4. L. J. Cutrona, "Synthetic aperture radar" in Radar Handbook, New York:McGraw-Hill, pp. 23.1-23.25, 1970.

5. C. Cafforio and C. Prati and F. Rocca, SAR data focusing using seismic migration techniques, IEEE Transactions on Aerospace and Electronic Systems, 1991, vol. 27, no 2, pp. 194-207, doi 10.1109/7.78293, ISSN 0018-9251

6. R. Bamler, A comparison of range-Doppler and wavenumber domain SAR focusing algorithms, IEEE Transactions on Geoscience and Remote Sensing, 1992, vol 30,no 4, pp. 706-713, doi10.1109/36.158864, ISSN 0196-2892

7. Golub, G. H. and Reinsch, C., Singular value decomposition and least squares solutions, Numerische Mathematik, 1970, Apr, 01, vol. 14, no.5, pp. 403-420, issn 0945-3245, doi 10.1007/BF02163027

8. A. S. Amein and J. J. Soraghan, Fractional Chirp Scaling Algorithm: Mathematical Model, IEEE Transactions on Signal Processing, 2007, vol 55, no 8, pp. 4162-4172, doi 10.1109/TSP.2007.895994, ISSN 1053-587X

9. W. Wang and R. Wang and Z. Zhang and Y. Deng and N. Li and L. Hou and Z. Xu, First Demonstration of Airborne SAR With Nonlinear FM Chirp Waveforms, IEEE Geoscience and Remote Sensing Letters, 2016, Feb, vol. 13, no. 2, pp. 247-251, doi: 10.1109/LGRS.2015.2508102, ISSN: $1545-598 X$ 
10. P. Imperatore and A. Pepe and R. Lanari, Spaceborne Synthetic Aperture Radar Data Focusing on Multicore-Based Architectures, IEEE Transactions on Geoscience and Remote Sensing, 2016, Aug, vol. 54, no. 8, pp. 4712-4731, doi: 10.1109/TGRS.2016.2550201, ISSN: 0196-2892

11. L. Chen and D. An and X. Huang, Extended Autofocus Backprojection Algorithm for Low-Frequency SAR Imaging, IEEE Geoscience and Remote Sensing Letters, 2017, Aug, vol. 14, no. 8, pp. 1323-1327, doi: 10.1109/LGRS.2017.2711005, ISSN: 1545-598X

12. L. Ran and Z. Liu and L. Zhang and T. Li and R. Xie, An Autofocus Algorithm for Estimating Residual Trajectory Deviations in Synthetic Aperture Radar, IEEE Transactions on Geoscience and Remote Sensing, 2017, June, vol. 55, no. 6, pp. 3408-3425, doi: 10.1109/TGRS.2017.2670785, ISSN: 0196-2892

13. D. Li and H. Lin and H. Liu and G. Liao and X. Tan, Focus Improvement for High-Resolution Highly Squinted SAR Imaging Based on 2-D Spatial-Variant Linear and Quadratic RCMs Correction and Azimuth-Dependent Doppler Equalization, IEEE Journal of Selected Topics in Applied Earth Observations and Remote Sensing, 2017, Jan, vol. 10, no. 1, pp. 168-183, doi: 10.1109/JSTARS.2016.2569561, ISSN: 1939-1404

14. J. Ma and H. Tao and P. Huang, Subspace-based super-resolution algorithm for ground moving target imaging and motion parameter estimation, IET Radar, Sonar Navigation, 2016, vol. 10, no. 3, pp. 488-499, doi: 10.1049/iet-rsn.2015.0188, ISSN: 1751-8784

15. J. Torgrimsson and P. Dammert and H. Hellsten and L. M. H. Ulander, An Efficient Solution to the Factorized Geometrical Autofocus Problem, IEEE Transactions on Geoscience and Remote Sensing, 2016, Aug, vol. 54, no. 8, pp. 4732-4748, doi: 10.1109/TGRS.2016.2550663, ISSN: 0196-2892

16. Brian D. Rigling, Flying blind: a challenge problem for SAR imaging without navigational data, Proc. SPIE, 3 May 2012, vol. 8394, doi 10.1117/12.923439

17. L. Zhang and M. Hu and G. Wang and H. Wang, Range-Dependent Map-Drift Algorithm for Focusing UAV SAR Imagery, IEEE Geoscience and Remote Sensing Letters, 2016, Aug, vol. 13, no. 8, pp. 1158-1162, doi: 10.1109/LGRS.2016.2574752, ISSN: 1545-598X

18. P. Guo and S. Tang and L. Zhang and G. C. Sun, Improved focusing approach for highly squinted beam steering SAR, IET Radar, Sonar Navigation, 2016, vol. 10, no. 8, pp. 1394-1399, doi: 10.1049/iet-rsn.2015.0473, ISSN: 1751-8784

19. Z. Li and Y. Liang and M. Xing and Y. Huai and L. Zeng and Z. Bao, Focusing of Highly Squinted SAR Data With Frequency Nonlinear Chirp Scaling, IEEE Geoscience and Remote Sensing Letters, 2016, Jan, vol. 13, no. 1, pp. 23-27, doi: 10.1109/LGRS.2015.2492681, ISSN: 1545-598X

20. C. Noviello and G. Fornaro and M. Martorella, Focused SAR Image Formation of Moving Targets Based on Doppler Parameter Estimation, IEEE Transactions on Geoscience and Remote Sensing, 2015, June, vol. 53, no. 6, pp. 3460-3470, doi: 10.1109/TGRS.2014.2377293, ISSN: 0196-2892

21. S. Tang and L. Zhang and P. Guo and G. Liu and Y. Zhang and Q. Li and Y. Gu and C. Lin, Processing of Monostatic SAR Data With General Configurations, IEEE Transactions on Geoscience and Remote Sensing, 2015, Dec, vol. 53, no. 12, pp. 6529-6546, doi: 10.1109/TGRS.2015.2443835, ISSN: 0196-2892

22. C. Guaragnella, T. D'Orazio, B.SAR - Blind SAR Data Focusing, SPIE - International Conference on Image and Signal Processing for Remote Sensing, Berlin, 2018, sept. 10-13 